\title{
Continuous positive airway pressure delivery during less invasive surfactant administration: a physiologic study
}

\author{
Gilles Jourdain $^{1} \cdot$ Marie De Tersant $^{1} \cdot$ Valentina Dell'Orto $^{1} \cdot$ Giorgio Conti $^{1,2} \cdot$ Daniele De Luca $^{1,2}$
}

Received: 6 April 2017 / Accepted: 25 October 2017 / Published online: 1 December 2017

(c) Nature America, Inc., part of Springer Nature 2018

\begin{abstract}
Objective We sought to investigate the pressure delivery during less invasive surfactant administration, as we hypothesize that it might be reduced.

Study design Physiologic in vitro study in a ventilation lab, using different pressure generators, levels, and leaks in a model of neonatal airways/lung mimicking mechanical characteristics of respiratory distress syndrome. Pressure was measured at the lung and verified in vivo measuring pharyngeal pressure in 19 neonates under same conditions. Data were analyzed using repeated measures-analysis of variance.

Results Pressure delivery in vitro is significantly and variably reduced during minimally invasive surfactant administration: pressure loss is $\approx 99 \%$ and $\approx 10-97 \%$, during mouth opening and closure, respectively. Pressure loss seems independent from the type of CPAP and interface. In vivo measurements showed similar pressure drops.

Conclusions Pressure transmission during minimally invasive surfactant administration is significantly reduced or totally absent. Pressure drop occurs despite the increased airway resistances and the airflow limitation due to the tracheal catheterization, but is independent from the type of pressure generator and interface.
\end{abstract}

\section{Introduction}

Early nasal continuous positive airway pressure (CPAP) is now recognized as the cornerstone treatment of respiratory distress syndrome (RDS) in preterm neonates. Both European and American guidelines advise so [1, 2], since avoiding mechanical ventilation has proven to be beneficial according to meta-analyses of several randomized controlled trials $[3,4]$. For CPAP to be maximally efficacious, interfaces should be carefully chosen $[5,6]$ and pressure interruptions should be minimized avoiding lung derecruitment [7].

Electronic supplementary material The online version of this article (https://doi.org/10.1038/s41372-017-0009-3) contains supplementary material, which is available to authorized users.

$\checkmark$ Daniele De Luca

dm.deluca@icloud.com

1 Division of Pediatrics, Neonatal Critical Care and Transportation, Medical Center "A.Béclère", South Paris University Hospitals, APHP, Paris, France

2 VentiLab, Institute of Anesthesiology and Critical Care, Catholic University of the Sacred Heart, Rome, Italy
However, CPAP failure may still occur and patients may require surfactant administration. Surfactant may be administered after intubation through the intubation-surfactantextubation method, which requires an endotracheal tube placement under laryngoscopy [8]. An alternative procedure using thin catheters unsuitable to ventilate has been recently proposed: this procedure still requires laryngoscopy and has been described in two slightly different variants and they have been given the label "minimally invasive surfactant therapy" or "less invasive surfactant administration." The first variant uses a stiff vascular catheter (usually inserted through the mouth), while the second needs a soft feeding tube (usually inserted through a nostril and guided with a McGill's forceps) [8]. The main advantages of these techniques are supposed to be the reduced invasivity, the avoidance of mechanical ventilation and the continuation of CPAP during surfactant administration [8]. However, no data are available about the CPAP stability and the actual pressure delivery during surfactant administration with these techniques. In some cases, nasal prongs were kept in place; in some others, they were removed during the procedure $[9,10]$. Moreover, it is not always specified if patient's mouth is left open or closed during surfactant administration $[9,10]$ and different CPAP generators and interfaces may be used [11]: all these factors might impact on pressure delivery. 
We sought to investigate the pressure delivery during less invasive surfactant administration, as we hypothesized that it might be significantly reduced. Thus, we performed an in vitro study with different types of CPAP systems, interfaces and degree of leaks; then, we verified the pressure drop in a series of clinically stable neonates under the same conditions.

\section{Methods}

\section{In vitro model}

A neonatal mannequin with upper airways mimicking a preterm infant (Laerdal inc., Stavanger, Norway) has been used. Its trachea was cut and tightly connected to a newborn lung model (n.20/01/0082, Acutronic Medical System, Zurich, Switzerland), as previously published [12]. The mannequin and the lung reproduced the mechanical characteristics of RDS in preterm neonates (compliance: 0.4 $\mathrm{mL} / \mathrm{cm} \mathrm{H}_{2} \mathrm{O}, 3 \mathrm{~cm}$ tracheal length, $3 \mathrm{~mm}$ internal tracheal diameter [13, 14]). A respiratory function monitor (FLORIAN®, Acutronic Medical System, Zurich, Switzerland) was connected to the lung model through a low compliance line and a stopcock to measure the pressure at the lung $\left(P_{\text {lung }}\right)$. The FLORIAN@ pressure transducer has been specifically designed for neonatal ventilation and has an accuracy of $\pm 4 \%$, as per its technical details (Acutronic Medical System, Zurich, Switzerland). The model was connected to either a variable flow device (VF-CPAP: Infant Flow ${ }^{\circledR}$, Carefusion, San Diego, CA, USA) or to a continuous flow ventilator (CF-CPAP: BabyLog $8000+\circledR$, Drager, Lubeck, Germany), through dedicated circuits and appropriately sized nasal mask (nFLOW® Nasal Mask Large, Intersugical Ltd. Wokingham, UK and FlexyTrunk® Nasal Mask Large, Fisher\&Paykel Healthcare, Auckland, New Zealand, for VF-CPAP and CF-CPAP, respectively). We used these masks as they resulted superior to nasal prongs [6] and they represent the first line interface used in our neonatal intensive care unit (NICU). Before the study, these devices underwent a technical revision and have been set as per the manufacturer's recommendations.

\section{Protocol}

Preliminary tests were performed with both types of CPAP generators. CPAP was progressively set at 4, 5, and 6 $\mathrm{cmH}_{2} \mathrm{O}$, the circuit was directly connected to the test lung and secured to completely avoid leaks: CPAP was measured at the lung to demonstrate the accuracy of pressure transducer, against the CPAP value shown by the CPAP generator. A second test was then performed to verify model suitability: the circuit was connected to the mannequin and its mouth was closed using an investigator's hand. The bench model and the test are described in an illustrative video in the Supplementary electronic information-1. Each test lasted $15 \mathrm{~min}$ and no malfunctioning was noticed. Before each test the transducer was calibrated at zero pressure in room air.

The experimental procedure was divided in the following time-point. $T_{0}$ : before laryngoscopy and surfactant administration (mannequin mouth was closed during this phase); after $T_{0}$, laryngoscopy was performed and $P_{\text {lung }}$ was always zero during this procedure, thus it was not considered for study purposes. $T_{1}$ : surfactant administration with catheter placed into the trachea (and mouth either open or closed). $T_{2}$ : catheter withdrawal (achieved over $5 \mathrm{~s}$, while maintaining the mouth closed). $P_{\text {lung }}$ was recorded for the three timepoints; pressure tracing was continuously observed on the FLORIAN® screen and, if $P_{\text {lung }}$ was changing during $T_{1}$ and $T_{2}$, all values were recorded and averaged. Before each experiment, the transducer was checked at zero pressure in room air.

Three neonatal consultants skilled for less invasive surfactant administration performed the procedure three times for each type of CPAP and once per each CPAP level (4, 5, and $6 \mathrm{cmH}_{2} \mathrm{O}$ ); each procedure was repeated twice: one closing the mannequin mouth during $T_{1}$ and the second leaving the mouth open. Thus, a total of 12 (6 for CF-CPAP and 6 for VF-CPAP) procedures (for a total of 36 data points) have been performed and analyzed. Surfactant administration was performed as described elsewhere [10]. In detail, a soft 4Fr feeding catheter (Vygon, Ecouen, France) was placed, by a Magill's forceps, under laryngoscopy using a Miller's blade 0. A 2 kg-neonate was mimicked and $5 \mathrm{~mL}$ of air (equivalent to the volume of 200 $\mathrm{mg} / \mathrm{kg}$ poractant- $\alpha$ ) were slowly injected over $20 \mathrm{~s}$ [9]. An investigator measured the time needed for each procedure and filmed it: videos were reviewed later and no errors were noticed. An illustrative video showing the model and one procedure is available in the electronic Supplementary informations-2. Since some NICUs may prefer to use nasal prongs over masks and, given the results obtained in the first series of experiments, we repeated the measurements in $T_{1}$ using different nasal prongs and both closing and keeping the mouth open. nFLOW® Nasal Prongs Large and Medium (Intersugical Ltd. Wokingham, UK) were used for VF-CPAP. FlexyTrunk® Nasal Prongs 50/50 and 30/20 (Fisher\&Paykel Healthcare, Auckland, New Zealand) and RAM Cannula ${ }^{\circledR}$ micropremie and infant (NeoTech, Valencia, CA, USA) were tested for CF-CPAP.

\section{In vivo measurements}

Following in vitro experiments, the pressure drop (in \%) was calculated as follows: (CPAP- $\left.P_{\text {lung }}\right) / \mathrm{CPAPx} 100$; 
CPAP was considered as the CPAP level shown on the ventilator screen while measuring $P_{\text {lung. }}$. We wanted to verify if a similar pressure drop was occurring in vivo in CPAP-treated, stable, preterm neonates with RDS. To do this, we measured pharyngeal pressure $\left(P_{\text {phar }}\right)$ using the same FLORIAN® monitor connected to a 6 Fr polyurethane air-filled sterile catheter gently positioned in the oropharynx, as previously described [15]. The catheter was left in place for $5 \mathrm{~min}$, pressure tracing was continuously observed, pressure value was recorded at $30 \mathrm{~s}$ intervals, both during inspiration and expiration and measures were averaged. If pressure tracing presented artefacts due to movements, swallowing or catheter displacement, the catheter was changed and the measurement repeated. Before each measurement the transducer was checked at zero pressure in room air. The measurements were done when the neonate was in a quiet state, after the routine removal of oral secretions and both during mouth opening and closure. Active mouth closure was achieved with gentle pressure under the patients' chin with a finger. A minimum sample size of six babies per each type of CPAP was required, considering a mean pressure drop of 40 (18.7)\% between measurements with closed and open mouth, as previously described [15]. An $\alpha$-error of 0.05 and a power of $90 \%$ were set. We finally recruited eleven and eight neonates for the measurements in VF-CPAP and CF-CPAP, respectively, as this was a sample size comparable to that of other studies in the field [15, 16]. In vivo pressure drop was calculated as described above, replacing $P_{\text {lung }}$ with $P_{\text {phar }}$ and using the CPAP level shown on the ventilator screen while measuring $P_{\text {phar }}$. According to our internal clinical protocol, in our NICU all neonates with RDS are treated with either VFCPAP or CF-CPAP if they were $\leq$ or $>32$ weeks' gestation, respectively [17]. This choice aims to reduce as much as possible the work of breathing in the most preterm neonates [11]. Measurements have been performed during the routine nursing care, which includes aspiration of upper airway secretions, thus with no change in clinical routine care. Measurements lasted a maximum of 2 min per baby. Ethical committee approved these measurements and oral informed consent were obtained from parents.

\section{Statistics}

Data were expressed as mean (standard deviation). $P_{\text {lung }}$ within the same procedures was analyzed with repeated measures-analysis of variance across the three timepoints followed by Bonferroni post-hoc comparisons. $P_{\text {lung }}$ and pressure drop have been compared by Student paired $t$-test between the experiments performed with CF-CPAP or VFCPAP, those with open or closed mouth and those with different types of nasal interfaces. CPAP and $P_{\text {phar }}$ during mouth opening and closure have also been analyzed with
Student paired $t$-test. Statistics have been performed with MedCalc rel.13.3 (MedCalc bvba, Ostend, Belgium) and $p$ $<0.05$ has been considered as statistically significant.

\section{Results}

$P_{\text {lung }}$ in the bench model significantly varies during minimally invasive surfactant administration when the mouth is open, both using VF-CPAP (overall $p<0.0001$ ) and CFCPAP (overall $p<0.0001$ ); post-hoc comparisons are shown in Fig. 1a. Pressure drop at $T_{1}$ was 99.7 (3)\% for both VF-CPAP and VF-CPAP, while at $T_{2}$ it was 46.7 (38)\% and 48.4 (17.5)\% for VF-CPAP and CF-CPAP, respectively.

$P_{\text {lung }}$ in the bench model also significantly changes when the mouth is closed, both using VF-CPAP (overall $p<$ 0.0001) and CF-CPAP (overall $p<0.0001$ ); post-hoc comparisons are shown in Fig. 1b. Pressure drop at $T_{1}$ was 25.9 (17)\% and 20 (24)\% for VF-CPAP and CF-CPAP, respectively, while at $T_{2}$ it was $43.6(30.3) \%$ and 69.2 (33.8)\%, for VF-CPAP and CF-CPAP, respectively. At $T_{1}$, $P_{\text {lung }}$ is lower and pressure drop is greater for the experiments with the open mouth than for those with the closed mouth $(p<0.0001$ for both measurements done with VFCPAP and CF-CPAP). $P_{\text {lung }}$ and pressure drop is not significantly different between VF-CPAP and CF-CPAP at any time-point (data not shown). The bench procedure lasted on average $70(20) \mathrm{s}$; no problems occurred and there were no differences between the procedures performed by the three operators.

Experiment with different types of nasal prongs and open mouth showed a pressure drop at $T_{1}$ of 98.9 (1.9)\% for all types of prongs, both in VF-CPAP and CF-CPAP; these findings were not different from those obtained with nasal masks $(p=0.443)$. Measurements with nasal prongs and closed mouth showed, during VF-CPAP, a $T_{1}$-pressure drop of 15.3 (8)\% and 20.5 (4.2)\% with large and medium prongs, respectively; these findings were not different from those obtained with nasal masks $(p=0.06$ and $p=0.297$ for large and medium prongs, respectively). During CFCPAP, pressure drop at $T_{1}$ was $28.3 \%$ (12.6)\% with both Flexytrunk® 50/50 and 30/20 prongs and 29.5 (4.2)\% with RAMCannula ${ }^{\circledR}$ infant-size; these findings were not different from those obtained with nasal masks $(p=0.3$ and $p=$ 0.190 for the two types of prongs, respectively). Pressure drop was 97.2 (4.8)\% with RAMCannula ${ }^{\circledR}$ micropremiesize; this result was significantly higher than the value obtained with nasal masks $(p=0.0001)$.

In vivo measurements and basic population details are shown in the Table 1.

$P_{\text {phar }}$ during mouth opening was significantly lower than during mouth closure, both for VF-CPAP $(p=0.0009)$ and 

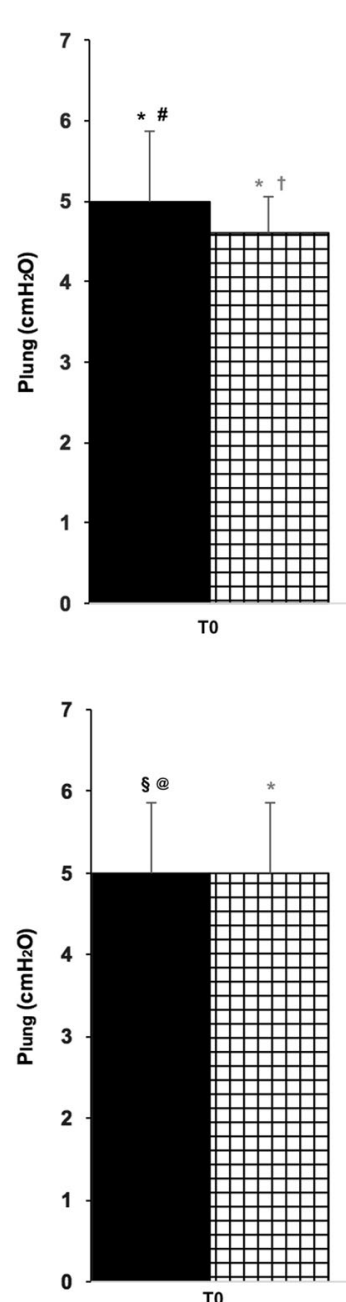

Fig. 1 In vitro study of pressure transmitted at the lung during minimally invasive surfactant administration. Black and hatched columns represent mean pressure transmitted at the lung level $\left(P_{\text {lung }}\right)$ with variable (VF-CPAP) and continuous (CF-CPAP) flow continuous positive airway pressure, respectively. T-bars represent standard deviations. $T_{0}$ and $T_{2}$ measurements have been performed with mouth closure; $T_{1}$ measurements have been performed with open (panel a) or closed (panel b) mouth. Black and gray symbols represent post-hoc comparisons within the same setting, for VF-CPAP and CF-CPAP,

CF-CPAP $(p=0.0091) . P_{\text {phar }}$ was significantly lower than the set CPAP only when the patients' mouth was open $(p=$ 0.0008 for VF-CPAP and $p=0.015$ for CF-CPAP). During VF-CPAP pressure drop was $53(7.8) \%$ when the patients' mouth was open and $1.5(1.5) \%$ when it was closed; using CF-CPAP pressure drop was 47 (15.6)\% during mouth opening and 9.3 (11)\% during mouth closure. $P_{\text {phar }}$ was not significantly different between neonates receiving VFCPAP or CF-CPAP. All neonates were clinically stable with an oxygen saturation $>90 \%$ and inspired oxygen fraction $\leq 0.25$ and no changes in any vital parameters occurred despite the variation in pressure transmission.
A
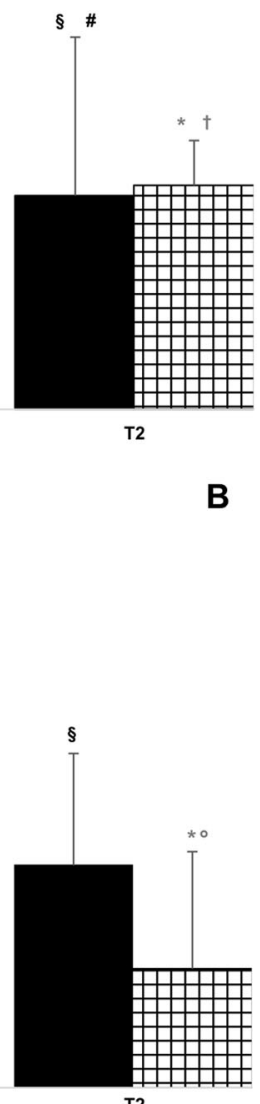

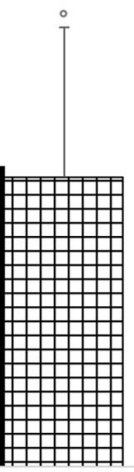

respectively. More details in the text. a (open mouth): Bonferroni posthoc comparisons for experiments performed with VF-CPAP $\left({ }^{*} p<\right.$ $0.0001 ;{ }^{\#} p=0.02 ;{ }^{8} p=0.01$ ); Bonferroni post-hoc comparisons for experiments performed with CF-CPAP $\left(* p<0.0001 ;{ }^{\dagger} p=0.0009\right)$. $\mathbf{b}$ (closed mouth): Bonferroni post-hoc comparisons for experiments performed with VF-CPAP $\left({ }^{\S} p=0.01 ;{ }^{\circledR} p=0.008\right)$; Bonferroni posthoc comparisons for experiments performed with CF-CPAP $\left({ }^{*} p<\right.$ $\left.0.0001 ;{ }^{\circ} p=0.003\right)$. CPAP continuous positive airway pressure, $P_{\text {lung }}$ pressure at the lung model

\section{Discussion}

We demonstrated that CPAP transmission during minimally invasive surfactant administration may be significantly reduced or totally absent. Recent meta-analyses suggest a possible improvement in clinical outcomes using minimally invasive surfactant administration techniques, but the available data seem limited by the overall low quality and lack of robustness [18, 19]. Moreover, clear physiopathological data explaining the possible clinical improvement are lacking: a main suggested explanation would be the avoidance of mechanical ventilation and its negative consequences for the 
Table 1 Population details and in vivo measurements

\begin{tabular}{|c|c|c|}
\hline Neonates & $\begin{array}{l}\text { VF-CPAP } \\
11\end{array}$ & $\begin{array}{l}\text { CF-CPAP } \\
8\end{array}$ \\
\hline Gestational age (weeks) & $28.8(3.6)$ & $33.6(0.5)$ \\
\hline Birth weight (grams) & $1309(841)$ & $1750(395)$ \\
\hline Postnatal age (days) & $3(0.8)$ & $2(0.5)$ \\
\hline Male sex & $6(54.5 \%)$ & $4(50 \%)$ \\
\hline Set CPAP $\left(\mathrm{cmH}_{2} \mathrm{O}\right)$ & $6.2(2)^{\S}$ & $6.3(1.6)^{\circ}$ \\
\hline$P_{\text {phar }}$ with closed mouth $\left(\mathrm{cmH}_{2} \mathrm{O}\right)$ & $6.2(2.7)^{*}$ & $5.6(1.6)^{\#}$ \\
\hline$P_{\text {phar }}$ with open mouth $\left(\mathrm{cmH}_{2} \mathrm{O}\right)$ & $2.9(1.9)^{*, \S}$ & $3.3(1.4)^{\#, \circ}$ \\
\hline
\end{tabular}

Data are expressed as mean (standard deviation) or number $(\%)$. Babies were receiving CPAP through appropriately sized nFLOW® Nasal Mask (Intersugical Ltd. Wokingham, UK) and FlexyTrunk® Nasal Mask (Fisher\&Paykel Healthcare, Auckland, New Zealand), for VF-CPAP and CF-CPAP, respectively

$C P A P$ continuous positive airway pressure, $P_{\text {phar }}$ pharyngeal pressure ${ }^{*} p=0.0009 ;{ }^{\#} p=0.0091 ;{ }^{\S} p=0.0008 ;{ }^{\circ} p=0.015$ with Student paired $t$-test

preterm lung [8]. The continued transmission of CPAP during surfactant administration is regarded as an important mechanism to avoid invasive ventilation, since CPAP would promote alveolar recruitment and surfactant diffusion [8]. However, until now there was a lack of physiological studies about pressure transmission and lung mechanics during less invasive surfactant administration.

Our findings provide data partially filling this gap. CPAP is not well transmitted during minimally invasive surfactant administration, as a significant reduction in $P_{\text {lung }}$ is observed. The pressure drop is clearly higher when the procedure is performed during mouth opening. However, a pressure drop is still evident and presents considerable variations also when the procedure is performed during mouth closure. De Paoli et al. [15] reported roughly similar and significant pressure drops in vivo. This effect is clearly due to the leak variations occurring around nasal interfaces. Moreover, air leaks from between the lips might also occur despite jaw closure, especially when pulling out the catheter: this may explain the variable pressure drops in $T_{2}$, that is, during catheter withdrawal with mouth closure. Our findings seem independent from the type of nasal interface, as similar and variable pressure drops have been demonstrated with nasal mask or different types and sizes of binasal prongs. The worst performance is achieved by the smallest RAMCannula ${ }^{\circledR}$, which is not surprising. In fact, RAMCannula ${ }^{\circledR}$ are supposed not to fill the nares diameter and this leads to a relevant pressure drop as previously published [20, 21].

Whether or not this loss may impact on relevant clinical outcomes remains to be determined, but similar pressure drops have been associated with lung derecruitment in vivo [7]. $P_{\text {phar }}$ measurements demonstrated that in vitro data reliably reproduced the pressure transmission observed in the actual clinical setting. Moreover, our data are consistent with those reported by other authors who demonstrated similar pressure drops $[15,16,22]$. A nebulized surfactant may clearly overcome the problem as it will not influence at all CPAP transmission.

The insertion of a non-ventilated catheter into the trachea may have profound effects on lung mechanics and gas exchange: this issue has been deeply studied in adults with hypoxemic respiratory failure undergoing diagnostic fibrobronchoscopy [23-25]. Hypoxia and hypercapnia with variable recovering times have been reported in these patients [23, 24]. These effects are due to the insertion of an object in the airways, which unavoidably decreases the cross-sectional area available for the airflow, increasing airway resistances, especially during expiration [25]. This process could theoretically produce a certain amount of auto-PEEP, although we did not observe any pressure increment, but rather a pressure drop. This means that, despite the likely generation of auto-PEEP (which should increase the airway pressure beyond the set CPAP level), the leaks overcome this generation and the resulting airway pressure is lower than the set CPAP [24, 25]. The airway flow limitation due to an increase in resistances might be responsible for the transient hypoxemia/desaturations and bradycardias reported during minimally invasive surfactant administration [26]: in fact, adults undergoing bronchoscopy have experienced arrhythmias and desaturations [27]. Diagnostic bronchoscopy is a quick procedure if performed by expert operators (mean duration $\approx 5 \min [23,24]$ ), although slightly longer than minimally invasive surfactant administration. However, the airflow limitation is likely to be more relevant for neonates than for adults, given the higher respiratory rate and the smaller diameter of their airways. In fact, airway resistances are known to be directly proportional to the airway radius elevated to the fourth power (or the fifth power, in case of non-laminar flow), according to the Hagen-Poiseuille's law. Average tracheal diameter is 3 and $25 \mathrm{~mm}$ for preterm neonates and adults, respectively $[14,28]$; considering the use of $4 \mathrm{Fr}(\approx 1.35 \mathrm{~mm}$ diameter) feeding tube or a $4.2 \mathrm{~mm}(\approx 13 \mathrm{Fr})$ fibrobroncoscope in newborn and adult patients, respectively, we calculated the loss in cross-sectional area available for the airflow and the increment in airway resistances during tracheal catheterization (Fig. 2). The area available for the airflow is much lower in neonates than adults, while resistances in neonates are increased almost six times more than in adults. Resistances may be even higher during turbulent airflow, such as it occurs during crying. Only 36\% of neonates undergoing less invasive surfactant administration receive some forms of analgesia/sedation [29], thus turbulent airflow is more likely to occur and the airflow limitation mechanism is more relevant under these circumstances. The side effects of airflow limitation during bronchoscopy in 


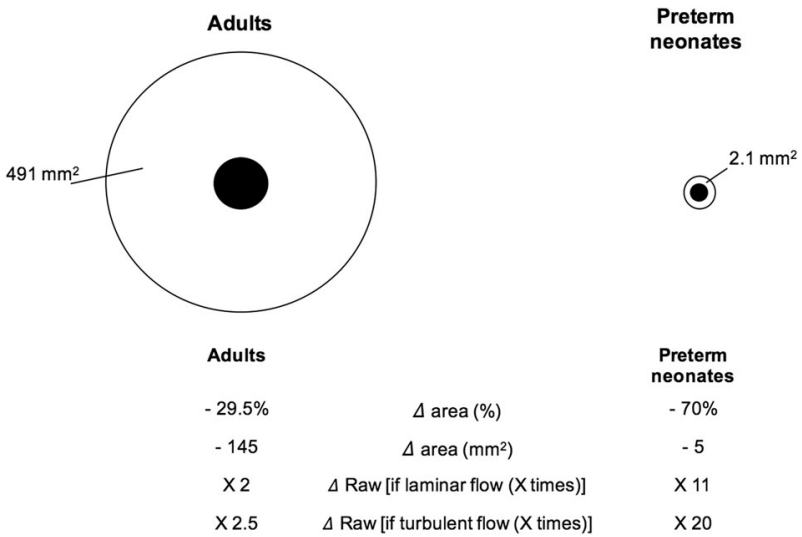

Fig. 2 Estimated airflow limitation during tracheal catheterization in adults and preterm neonates using a non-ventilated device. A fibrobronchoscope ( $4.2 \mathrm{~mm}$ diameter) and a feeding catheters used for less invasive surfactant administration $(1.35 \mathrm{~mm}$ diameter) are simulated. Tracheas and devices are represented by the outer and black inner circles, respectively; diameters are shown according to literature data $[14,28]$ and proportioned between them. Variation ( $\Delta$ area) in crosssectional area and in airway resistances $(\Delta$ Raw) during tracheal catheterization are reported. Numbers in the circles represent the crosssectional area available for the airflow; doing the calculation for the modified procedure using a vascular catheter $(1.7 \mathrm{~mm}$ diameter) would provide slightly worse $\Delta$ area and $\Delta$ Raw

adults have been overcome providing non-invasive ventilation (NIV) $[23,24]$ and a recent trial demonstrated a similar beneficial effect of NIV in preterm neonates undergoing minimally invasive surfactant administration [30].

We acknowledge some study limitations. The continued transmission of CPAP during surfactant administration could have been an explanation for the possible beneficial effect of less invasive surfactant administration techniques, but pressure transmission is unfortunately significantly reduced or zeroed. However, there might be other explanations. Our data help to understand physiology behind these techniques, but they are limited to that and do not intend to provide any definite conclusion on their clinical use. Our findings could be useful for designing future trials about surfactant administration techniques. We did not perform pressure measurements during less invasive surfactant administration in vivo. Thus, we cannot provide any data about the effect of in vivo sedation and the real surfactant administration although both could theoretically influence pressure transmission.

In fact, changes in intrathoracic pressure during inspiration may be impeded by partial airway obstruction with a viscous solution. Moreover, we just wanted to verify if our in vitro measurements during mouth opening or closure were reliable and they are actually consistent with in vivo and literature data $[15,16,22]$. We cannot provide any data about difficult intubations, the use of different catheters or other types of CPAP generators (e.g.: "bubble" CPAP), although we believe that these will lead to small differences, if any. Our model lacks of spontaneous breathing and generation of negative intrapleural pressure, but the pressure loss is unlikely to be influenced by the spontaneous breathing effort. Finally, we did not perform any experiment at higher levels of CPAP, since they are not used in our NICU protocol, which is based on the European guidelines [2] Higher CPAP level or the use of NIV might theoretically counterbalance the pressure loss, potentially reducing hypoxia and bradycardia [30] and may deserve to be studied further.

In conclusion, despite the reduction of the airway crosssectional area and the increased resistances, CPAP transmission during minimally invasive surfactant administration is significantly dropped or totally absent. There is virtually no pressure transmission if the procedure is performed with mouth opening; mouth closure provides a better transmission but still with a significant pressure drop. This effect is independent from the type of CPAP and nasal interface.

\section{Compliance with ethical standards}

Conflict of interest The authors declare that they have no conflict of interest.

\section{References}

1. Committee on Fetus and Newborn; American Academy of Pediatrics. Respiratory support in preterm infants at birth. Pediatrics. 2014;133:171-4.

2. Sweet DG, Carnielli V, Greisen G, Hallman M, Ozek E, Plavka R, et al. European Consensus Guidelines on the Management of Respiratory Distress Syndrome-2016 Update. Neonatology. 2017;111:107-125.

3. Schmölzer GM, Kumar M, Pichler G, Aziz K, O'Reilly M, Cheung PY. Non-invasive versus invasive respiratory support in preterm infants at birth: systematic review and meta-analysis. BMJ. 2013;347:f5980.

4. Fischer HS, Buhrer C. Avoiding endotracheal ventilation to prevent bronchopulmonary dysplasia: a meta-analysis. Pediatrics. 2013;132:e1351-60.

5. De Paoli AG, Morley CJ, Davis PG, Lau R, Hingeley E. In vitro comparison of nasal continuous positive airway pressure devices for neonates. Arch Dis Child Fetal Neonatal Ed. 2002;87:F42-5.

6. Kieran EA, Twomey AR, Molloy EJ, Murphy JF, O’Donnell CP. Randomized trial of prongs or mask for nasal continuous positive airway pressure in preterm infants. Pediatrics. 2012;130:e1170-6.

7. Courtney SE, Pyon KH, Saslow JG, Arnold GK, Pandit PB, Habib $\mathrm{RH}$. Lung recruitment and breathing pattern during variable versus continuous flow nasal continuous positive airway pressure in premature infants: An evaluation of three devices. Pediatrics. 2001;107:304-8.

8. Dargaville PA. Innovation in surfactant therapy I: Surfactant lavage and surfactant administration by fluid bolus using minimally invasive techniques. Neonatology. 2012;101:326-36.

9. Dargaville PA, Aiyappan A, Cornelius A, Williams C, De Paoli AG. Preliminary evaluation of a new technique of minimally invasive surfactant therapy. Arch Dis Child Fetal Neonatal Ed. 2011;96:F243-8.

10. Kribs A, Roll C, Göpel W, Wieg C, Groneck P, Laux R, et al. NINSAPP trial investigators: Nonintubated surfactant application 
vs conventional therapy in extremely preterm infants: A randomized clinical trial. JAMA Pediatr. 2015;169:723-30.

11. Pandit PB, Courtney SE, Pyon KH, Saslow JG, Habib RH. Work of breathing during constant- and variable-flow nasal continuous positive airway pressure in preterm neonates. Pediatrics. 2001;108:682-5.

12. De Luca D, Costa R, Visconti F, Piastra M, Conti G. Oscillation transmission and volume delivery during face mask-delivered HFOV in infants: Bench and in vivo study. Pediatr Pulmonol. 2016;51:705-12.

13. De Luca D, Carnielli VP, Conti G, Piastra M. Noninvasive high frequency oscillatory ventilation through nasal prongs: bench evaluation of efficacy and mechanics. Intensive Care Med. 2010;36:2094-100.

14. Harjeet M, Sahni D, Batra YK, Rajeev S. Anatomical dimensions of trachea, main bronchi, subcarinal and bronchial angles in fetuses measured ex vivo. Paediatr Anaesth. 2008;18:1029-34.

15. De Paoli AG, Lau R, Davis PG, Morley CJ. Pharyngeal pressure in preterm infants receiving nasal continuous positive airway pressure. Arch Dis Child Fetal Neonatal Ed. 2005;90:F79-81.

16. Colnaghi M, Matassa PG, Fumagalli M, Messina D, Mosca F. Pharyngeal pressure value using two continuous positive airway pressure devices. Arch Dis Child Fetal Neonatal Ed. 2008;93: F302-4.

17. Gizzi C, Klifa R, Pattumelli MG, Massenzi L, Taveira M, Shankar-Aguilera $\mathrm{S}$, et al. Continuous positive airway pressure and the burden of care for transient tachypnea of the neonate: Retrospective cohort study. Am J Perinatol. 2015;32:939-43.

18. Isayama $\mathrm{T}$, Iwami $\mathrm{H}$, McDonald $\mathrm{S}$, Bejene J. Association of noninvasive ventilation strategies with mortality and bronchopulmonary dysplasia among preterm infants: A systematic review and meta-analysis. JAMA. 2016;316:611-24.

19. Aldana-Aguirre JC, Pinto M, Featherstone RM, Kumar M. Less invasive surfactant administration versus intubation for surfactant delivery in preterm infants with respiratory distress syndrome: a systematic review and meta-analysis. Arch Dis Child Fetal Neonatal Ed. 2017;102:F17-23.
20. Mukerji A, Belik J. Neonatal nasal intermittent positive pressure ventilation efficacy and lung pressure transmission. J Perinatol. 2015;35:716-19.

21. Gerdes JS, Sivieri EM, Abbasi S. Factors influencing delivered mean airway pressure during nasal CPAP with the RAM cannula. Pediatr Pulmonol. 2016;51:60-9.

22. Chilton HW, Brooks JG. Pharyngeal pressures in nasal CPAP. J Pediatr. 1979;94:808-10.

23. Antonelli M, Conti G, Rocco M, Arcangeli A, Cavaliere F, Proietti R, et al. Noninvasive positive-pressure ventilation vs conventional oxygen supplementation in hypoxemic patients undergoing diagnostic bronchoscopy. Chest. 2002;121:1149-54.

24. Antonelli M, Conti G, Riccioni L, Meduri GU. Noninvasive positive pressure ventilation via face mask during bronchoscopy with BAL in high-risk hypoxemic patients. Chest. 1996;110:724-28.

25. Matsushima Y, Jones RL, King EG, Moysa G, Alton JD. Alterations in pulmonary mechanics and gas exchange during routine fiberoptic bronchoscopy. Chest. 1984;86:184-88.

26. More K, Sakhuja P, Shah PS. Minimally invasive surfactant administration in preterm infants: a meta-narrative review. JAMA Pediatr. 2014;168:901-8.

27. Goldstein RA, Rohatgi PK, Bergofsky EH, Block ER, Daniele RP, Dantzker DR, et al. ATS Board of Directors. Clinical role of bronchoalveolar lavage in adults with pulmonary disease. Am Rev Respir Dis. 1990;142:481-86.

28. McNiece WL, Dierdorf SF. The pediatric airway. Semin Pediatr Surg. 2004;13:152-65.

29. Mehler K, Oberthuer A, Haertel C, Herting E, Roth B, Goepel W. German Neonatal Network (GNN). Use of analgesic and sedative drugs in VLBW infants in German NICUs from 2003-2010. Eur J Pediatr. 2013;172:1633-9.

30. Oncel MY, Arayici S, Uras N, Alyamac-Dizdar E, Sari FN, Karahan S, et al. Nasal continuous positive airway pressure versus nasal intermittent positive-pressure ventilation within the minimally invasive surfactant therapy approach in preterm infants: a randomised controlled trial. Arch Dis Child Fetal Neonatal Ed. 2016;101:F323-8. 